\title{
Comparación del nivel de la cresta ósea en desdentados totales rehabilitados con implantes mandibulares y prótesis removible
}

\author{
Comparison of the level of the bone crest in total edentulous \\ rehabilitated with mandibular implants and removable prosthesis
}

\author{
Isabel Quiroga ${ }^{1 *}$, Catalina Dörner ${ }^{1}$, Marcial Sierra ${ }^{1}$, Julieta Del Pozo ${ }^{1}$, Rodrigo Quiroga ${ }^{1}$, Rodolfo Quiroga ${ }^{1}$
}

1. Universidad Mayor, Chile.

* Correspondencia autor: Isabel Quiroga Del Pozo. Asturias 171 oficina 102. Las condes. | Teléfono: +56981203671. | Email: quiro.isabe@gmail.com Trabajo recibido el 15/10/2017. Aprobado para su publicación el 20/01/2018

\begin{abstract}
RESUMEN
Objetivo: Se desarrolló una investigación que tuvo por propósito determinar el patrón de reabsorción del tejido óseo de implantes rehabilitados con coronas de acrílico que actúan como pilares de una prótesis parcial removible con retenedores extracoronarios. Materiales y métodos: Para medir tal efecto se diseñó un dispositivo que permitió estandarizar el registro del nivel óseo mediante una radiografía retroalveolar con técnica de paralelismo, considerando que este procedimiento permite observar gran cantidad de detalles, ofreciendo una imagen de alta definición, con una mínima distorsión, bajo costo y mínimo riesgo de radiación. Los resultados: indican que en un intervalo de seis meses medido después de realizada la rehabilitación a los 24 y 30 meses respectivamente se observa una reabsorción ósea que en promedio alcanza $0,12 \mathrm{~mm}$ siendo esta diferencia estadísticamente significativa entre ambos tiempos de registros. Conclusión: Los patrones de reabsorción de nuestra investigación son similares a los que ocurren con otras formas de rehabilitación sobre implantes oseointegrados
\end{abstract}

PALABRAS CLAVES

Nivel cresta ósea, Desdentados totales, Implantes mandibulares.

Rev. Clin. Periodoncia Implantol. Rehabil. Oral Vol. 11(1); 28-31, 2018.

\section{ABSTRACT}

Objetive: The purpose of this research was to determine the pattern of reabsorption of bone tissue from rehabilitated implants with acrylic crowns, acting as supports of a removable partial denture with extra-coronary retainers. Material and method: To measure this effect, a device was designed to standardize the measurement of the bone level by a retro-alveolar radiography with parallelism exposure technique, considering that this procedure allows to observe a large amount of details, offering a high definition image, with a minimum distortion, low cost and minimal risk of radiation. The results: indicate that in a six-month interval measured after rehabilitation at 24 and 30 months respectively, a bone resorption is observed, reaching an average of $0.12 \mathrm{~mm}$, this difference being statistically significant between both recording times. Conclusion: The resorption patterns of our research are similar to those that occur with other forms of rehabilitation on osseointegrated implants.

KEYWORDS

Bone crest level, Total edentulous, Mandibular implants.

Rev. Clin. Periodoncia Implantol. Rehabil. Oral Vol. 11(1); 28-31, 2018.

\section{INTRODUCCIÓN}

El tratamiento habitual a la condición de desdentado total es la prótesis completa removible, las que en general responden satisfactoriamente a los requerimientos funcionales en el maxilar superior, en cambio en la mandíbula, por razones anatómicas y fisiológicas condicionan un dispositivo con insuficientes propiedades de retención, soporte y estabilidad, lo que da origen a dificultades severas en la masticación y una permanente sensación de inseguridad para mantener la adaptación de la prótesis a los tejidos orales ${ }^{(1,2,3)}$. Como resultado de ello se afecta la autoestima, se limita la interacción social, compromete la nutrición, lo que se traduce en detrimento en la calidad de vida(4)

Considerando que nuestro país al igual que los países desarrollados, vive una acelerada transición hacia el envejecimiento demográfico de su población, donde se proyecta que para el año 2025, 1 de cada 5 individuos pertenecerá al grupo de adulto mayor ${ }^{(5)}$ y de acuerdo a los estudios realizados por el MINSAL, este grupo etario presenta un marcado deterioro de su salud oral (en Chile entre los 65-74 años de edad un $29,1 \%$ es desdentado total) ${ }^{(6)}$. Como consecuencia de ello se hace indispensable el desarrollo de nuevas formas de tratamientos que mejoren significativamente los niveles de satisfacción alcanzados con la prótesis total mandibular convencional.

En este contexto es que se desarrolló un proyecto de investigación FIDUM (Fondos de investigación de la Universidad Mayor) "Nuevo tratamiento a la condición de desdentado total mandibular, mediante una prótesis parcial retenida a dos implantes, para mejorar la calidad de vida del adulto mayor" consistente en transformar al desdentado total en 
desdentado parcial, mediante la construcción de dos coronas de acrílico cementadas a dos implantes de una fase colocados en la zona anterior de la mandíbula, para luego rehabilitar con prótesis parcial removible, retenidas en ambas coronas mediante retenedores extracoronarios. Modificando así el funcionamiento biomecánico, usando de soporte la mucosa masticatoria y retención a través de estos retenedores extracoronarios. Los resultados en relación a la eficacia masticatoria logrados con este nuevo tratamiento muestran un incremento comparable a la de individuos con dentadura natural, además de altos niveles de satisfacción ${ }^{(7)}$.

Continuando con esta línea de trabajo, es que hemos desarrollado una investigación que tiene como propósito evaluar el comportamiento del tejido óseo en relación a los implantes después de dos años de uso continuado de estos dispositivos.

Considerando que los retenedores ejercen sobre los pilares fuerzas eminentemente de carácter horizontal y que las coronas fueron confeccionadas en acrílico, ambas condiciones pueden influir en el patrón de reabsorción, la primera porque se desconoce el efecto sobre el implante de este tipo de carga y la segunda por el comportamiento del acrílico, en relación a la calidad de superficie y sello marginal de la corona, factores que pueden favorecer el desarrollo de cuadros de perimplantitis con los consecuentes efectos del deterioro sobre tejido óseo peri implantar.

Problema. Conocer o determinar el comportamiento del tejido óseo observado a nivel de la cresta marginal perimplantaria, cuando los implantes son rehabilitados con coronas confeccionadas en acrílico y éstas, actúan como pilares de una prótesis parcial removible recibiendo el esfuerzo biomecánico de retenedores extracoronarios.

\section{MATERIALES Y MÉTODOS}

Se realizó un estudio longitudinal y comparativo del patrón de reabsorción óseo crestal perimplantario. Las muestras se obtuvieron de 13 pacientes que participaron en el trabajo de investigación FIDUM "Nuevo tratamiento a la condición de desdentado total mandibular, mediante una prótesis parcial retenida a dos implantes, para mejorar la calidad de vida del adulto mayor". Este estudio se realizó en conformidad con los principios éticos de la declaración de Helsinki y fue revisado y aprobado por el comité de Ética de la Universidad Mayor.

Los pacientes fueron instruidos detalladamente de todos los procedimientos y propósitos de esta investigación, información redactada en el consentimiento informado firmado y autorizado por todos los participantes del estudio.

Se seleccionaron 25 implantes de una fase, rehabilitados con coronas periféricas confeccionadas con dientes de acrílico Marche ${ }^{\circledR}$, rebasados con acrílico de termocurado, cementados con vidrio ionómero a los muñones de los implantes oseointegrados instalados en la mandíbula de pacientes adultos mayores que participaron en el trabajo de investigación FIDUM del 2013. 5 hombres y 8 mujeres, cuyas edades fluctúan entre 58 y 78 años.

La variable dependiente fue el nivel de la cresta ósea perimplantaria (mesial y distal), de naturaleza cuantitativa, con grado de separación continua y nivel de medición intervalar. La variable independiente corresponde a un periodo de seis meses que transcurren después de realizada la primera medición, de naturaleza cuantitativa con grado de separación continua y nivel de medición intervalar.

Los pacientes fueron seleccionados según los siguientes criterios de inclusión:

- Uso permanente de la prótesis parcial removible, (proyecto FIDUM 2013) confeccionada en la facultad de odontología de la Universidad Mayor.

- Haber asistido a todos los controles del trabajo de investigación FIDUM "Nuevo tratamiento a la condición de desdentado total mandibular, mediante una prótesis parcial retenida a dos implantes, para mejorar la calidad de vida del adulto mayor".

- Los implantes deben ser considerados estables desde el punto de vista de la oseointegración.

Criterios de exclusión:

- Pacientes con características anatómicas que no permitieron aplicar o reproducir la técnica radiográfica del paralelismo.

Se usó porta films en todas las tomas radiográficas para permitir mantener la película paralela al eje longitudinal del implante y dirigir el rayo central perpendicular a ésta. Las prótesis parciales mandibulares de los pacientes, fueron replicadas en acrílico de autocurado y condicionadas para posicionar el porta films, una para el lado derecho y otra para el lado izquierdo. (fig1)

Se utilizaron 64 películas radiográficas Kodak ${ }^{\circledR}$ Ultra Speed para niños (tamaños $2 \times 3.5 \mathrm{~cm}$ ), equipo de radiografía análoga Minray (Soredex), posicionador paralelizador Mächtig, máquina de revelado Dürr Periomat

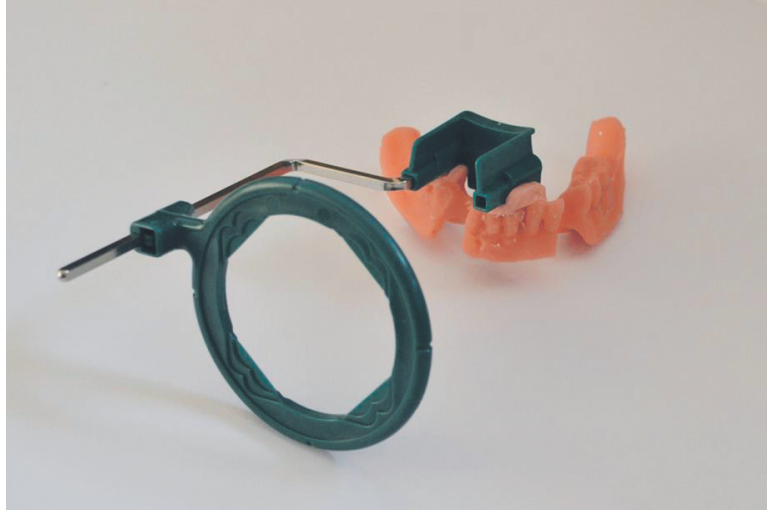

Figura 1. Dispositivo porta films y replica de prótesis removible.

1303-01 Dürr dental, escáner HCR dental digital X-ray Film Reader y software Handy Dentist 2.0.

Se tomaron 2 radiografías a cada implante, una a los 24 meses y otra a los 30 meses de instaladas las prótesis parciales removibles. Tiempo de exposición a $0.16 \mathrm{sg}$ y $70 \mathrm{Kv}$.

Se registró el nivel de las crestas óseas en las radiografías digitalizadas con el escáner HCR dental digital X-ray Film Reader, utilizando como puntos de referencia la parte superior del implante (muñón) y el tejido óseo más coronal en contacto con el implante, tanto por mesial como por distal. Posteriormente se utilizó el software Handy Dentist 2.0, para realizar las mediciones de las radiografías. (fig.2)

Los resultados fueron procesados y comparados a través del test $T$ pareado, valor de p 0,005, utilizando el software estadístico SYSTAT 14.1.

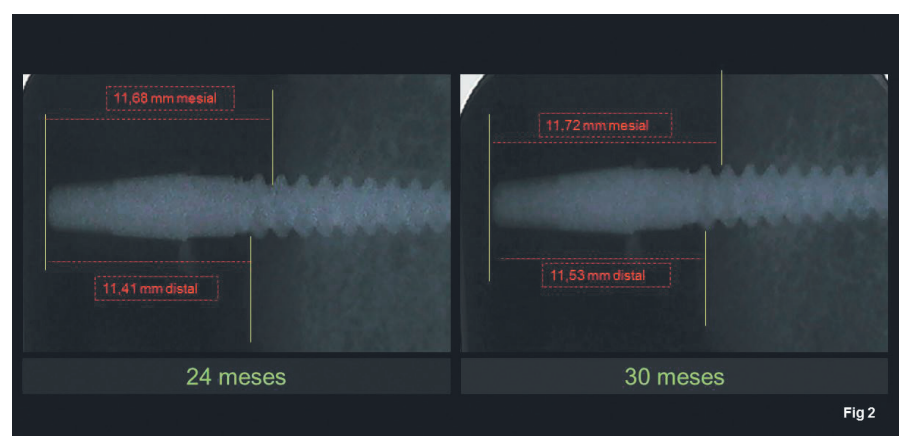

Figura 2. Radiografías digitalizadas donde se realizaron las mediciones.

\section{RESULTADOS}

Los resultados obtenidos al comparar las radiografías registradas a los 24 y 30 meses, mostraron una variación en el nivel de la inserción ósea, consistente en una pérdida de altura ósea perimplantaria de 0,12 $\mathrm{mm}$ en promedio. El análisis estadístico determinó que existe diferencia significativa con una desviación estándar de $0,11 \mathrm{~mm}$ y un valor de $p$ menor a 0,005. El valor máximo registrado al medir las radiografías del primer control fue de $12,85 \mathrm{~mm}$ siendo el mínimo de $9,56 \mathrm{~mm}$ y la mediana $10,59 \mathrm{~mm}$. En tanto, en el segundo control realizado 6 meses después observamos que el valor máximo fue de $13,21 \mathrm{~mm}$ y el valor mínimo de $9,68 \mathrm{~mm}$ siendo la mediana $10,69 \mathrm{~mm}$.

Los valores registrados se obtuvieron midiendo en la radiografía la distancia existente entre la parte superior del muñón del implante y la parte más coronal del tejido óseo en contacto con dicho dispositivo, tanto en mesial como en distal.

Las mediciones obtenidas por sitio y el promedio de ellos por implante a los 24 y 30 meses se expresan en la tabla $\mathrm{N}^{\circ} 1$.

En tabla $\mathrm{N}^{0} 2$ se compararon los promedios de las mediciones del nivel óseo registradas en las radiografías de los 25 casos a los 24 y 30 meses, analizado por el software Systat 14,1, con el test t pareado.

En el gráfico de cajas se evidencia la diferencia significativa que existe entre los promedios de las mediciones de cada implante en las radiografías obtenidas a los 24 y 30 meses. (fig.3)

Fig 3 : Diagrama de caja (vertical) para los datos de los promedios por implantes de las mediciones en radiografías.

En la tabla $\mathrm{N}^{\circ} 3$. Se exponen los valores de reabsorción ósea perimplantarias encontradas en las paredes mesial y distal de los 


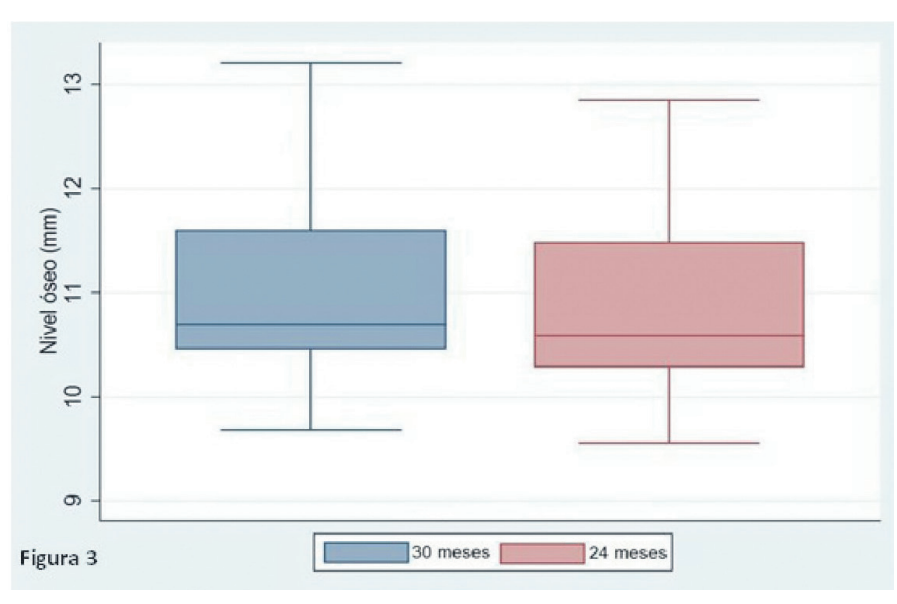

Figura 3. Diagrama de caja (vertical) para los datos de los promedios por implantes de las medicciones en radiografías

Tabla 2. Promedio de reabsorción ósea a los 24 y 30 meses.

\begin{tabular}{c|c|c|c}
\hline Variable & N de casos & Promedio & Std. Dev. \\
\hline 30 meses & 25 & 10,99 & 0,92 \\
24 meses & 25 & 10,87 & 0,91 \\
$\begin{array}{c}\text { Reabsorción ósea } \\
(\mathrm{mm})\end{array}$ & 25 & 0,12 & 0,11 \\
\hline \multicolumn{2}{c}{} & $\mathrm{t}=5,44$ & $\mathrm{p}<0,005$ \\
\cline { 2 - 4 }
\end{tabular}

implantes. Cabe señalar, que en 4 paredes se registraron ganancia en el nivel de inserción. La mayor pérdida observada fue de 0,36 mm.

\section{DISCUSIÓN}

Esta investigación surge de la necesidad de conocer el comportamiento del tejido óseo perimplantario frente a condiciones biomecánicas no estudiadas que difieren de la rehabilitación fija o sobredentaduras sobre implantes, en ambas formas de tratamiento se han cuantificado los patrones de reabsorción. Previo a esta investigación se desconocía el comportamiento del hueso al utilizar esta nueva técnica de rehabilitación.

Los resultados de esta investigación muestran que la dinámica del tejido óseo en torno a la pieza pilar conectada al implante tiene un comportamiento, en promedio, similar a lo que ocurre con las sobredentadura. El patrón de reabsorción óseo observado en el intervalo de 6 meses, fue de $0,12 \mathrm{~mm}$ de pérdida de inserción, considerando además que en 4 sitios, de los 50 testeados, se observa una aposición de tejido óseo.

Los patrones del comportamiento del tejido óseo en torno a los implantes de acuerdo a lo publicado por Gholami H. et al, Vercruyssen M. et $\mathrm{l}^{(8,9)}$ muestran que durante el periodo de seguimiento posterior al primer año de colocado los implantes y rehabilitados con sobredentaduras, se produce en promedio una reabsorción ósea de $0,2 \mathrm{~mm}$ anuales. Por otro lado cuando los implantes son rehabilitados de forma unitaria, la reabsorción ósea posterior al año es aproximadamente de $0,1 \mathrm{~mm}$ anual.

En general se reconoce que dos son los factores más significativos que inciden en patrones anormales de reabsorción ósea que ocurren en relación a un implante, la magnitud e incidencia de la fuerzas que actúan sobre la rehabilitación y el comportamiento del tejido gingival en torno al implante.

En relación a ello podemos señalar que las cargas masticatorias y demás requerimientos funcionales que operan sobre la prótesis se transmiten al implante a través del retenedor. En relación a ello podemos inferir que la magnitud y calidad de las fuerzas masticatorias en nuestra investigación no constituyen un factor de sobre esfuerzo funcional, considerando que la arcada antagonista es una prótesis total removible convencional. Otro factor que contribuye a los resultados obtenidos es que el soporte de la prótesis parcial es mucoso, por lo tanto los máximos esfuerzos mecánicos que operan sobre el diente pilar se suscitan durante la inserción y remoción del dispositivo protésico y no como ocurre en las sobredentaduras cuyo soporte es implanto-mucoso, condición que explicaría los fenómenos de báscula que de acuerdo a la literatura se describen con estos últimos dispositivos ${ }^{(10,11)}$.

Al examen visual de las piezas pilares no se observó cuadros inflamatorios en el entorno gingival, al parecer el acrílico, como material
Tabla 1. Mediciones por sitio y promedio de ellos por implantes los dos intervalos de tiempo.

\begin{tabular}{|c|c|c|c|c|c|}
\hline PACIENTE & Sitio & $\begin{array}{c}30 \\
\text { meses }\end{array}$ & $\begin{array}{c}\text { Prom_30 } \\
\text { meses }\end{array}$ & $\begin{array}{c}24 \\
\text { meses }\end{array}$ & $\underset{\text { meses }}{\text { Prom_24 }}$ \\
\hline \multirow{2}{*}{ Impl 1} & Mesial & 11,08 & \multirow{2}{*}{11,03} & 11,07 & \multirow{2}{*}{10,98} \\
\hline & Distal & 10,97 & & 10,88 & \\
\hline \multirow{2}{*}{ Impl 2} & Mesial & 11,72 & \multirow{2}{*}{11,63} & 11,68 & \multirow{2}{*}{11,55} \\
\hline & Distal & 11,53 & & 11,41 & \\
\hline \multirow{2}{*}{ Impl 3} & Mesial & 9,19 & \multirow{2}{*}{9,69} & 9,16 & \multirow{2}{*}{9,60} \\
\hline & Distal & 10,18 & & 10,04 & \\
\hline \multirow{2}{*}{ Impl 4} & Mesial & 10,24 & \multirow{2}{*}{10,10} & 10,17 & \multirow{2}{*}{10,05} \\
\hline & Distal & 9,95 & & 9,93 & \\
\hline \multirow{2}{*}{ Impl 5} & Mesial & 10,73 & \multirow{2}{*}{10,58} & 10,66 & \multirow{2}{*}{10,47} \\
\hline & Distal & 10,42 & & 10,28 & \\
\hline \multirow{2}{*}{ Impl 6} & Mesial & 12,13 & \multirow{2}{*}{12,62} & 12,08 & \multirow{2}{*}{12,56} \\
\hline & Distal & 13,10 & & 13,03 & \\
\hline \multirow{2}{*}{ Impl 7} & Mesial & 10,75 & \multirow{2}{*}{10,95} & 10,58 & \multirow{2}{*}{10,86} \\
\hline & Distal & 11,15 & & 11,14 & \\
\hline $1 \mathrm{mnl} / 8$ & Mesial & 13,21 & 1321 & 12,85 & 1285 \\
\hline о & Distal & 13,21 & 10,21 & 12,85 & 12,00 \\
\hline$|m n| 9$ & Mesial & 11,03 & 1100 & 11,03 & 1096 \\
\hline ك & Distal & 10,97 & 年, & 10,89 & ט, \\
\hline Imp 10 & Mesial & 12,64 & 1280 & 12,58 & 1261 \\
\hline Impl iv & Distal & 12,95 & & 12,63 & $|2,0|$ \\
\hline Impl 11 & Mesial & 11,37 & 10.51 & 11,36 & 1039 \\
\hline 1 & Distal & 9,65 & 10,51 & 9,42 & 10,09 \\
\hline $1 \mathrm{~mol} 12$ & Mesial & 10,53 & 1013 & 10,49 & 1060 \\
\hline 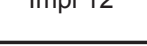 & Distal & 10,32 & 10,40 & 10,70 & (10,00 \\
\hline $1 \mathrm{~mol} 12$ & Mesial & 11,05 & 1070 & 11,00 & 1054 \\
\hline 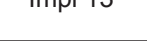 & Distal & 10,34 & 10,10 & 10,07 & 10,04 \\
\hline Imnl 14 & Mesial & 10,51 & 1071 & 10,53 & 1067 \\
\hline $1 \mathrm{mpl} 14$ & Distal & 10,90 & $10, \pi 1$ & 10,80 & 10,01 \\
\hline Impl 15 & Mesial & 12,56 & 1207 & 12,46 & 1200 \\
\hline 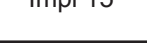 & Distal & 11,57 & 12,01 & 11,54 & 12,00 \\
\hline Impl 16 & Mesial & 9,65 & 978 & 9,23 & 956 \\
\hline 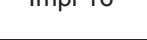 & Distal & 9,90 & , & 9,89 & 0,00 \\
\hline Impl 17 & Mesial & 10,35 & 1037 & 10,01 & 1003 \\
\hline 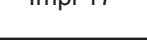 & Distal & 10,39 & 10,01 & 10,05 & ש \\
\hline Impl 18 & Mesial & 10,36 & 10.55 & 10,09 & 1026 \\
\hline 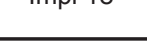 & Distal & 10,74 & 0,00 & 10,42 & (2) \\
\hline Impl 19 & Mesial & 11,99 & 1059 & 11,73 & 1043 \\
\hline & Distal & 9,18 & & 9,13 & \\
\hline $\mid \mathrm{mnl} 20$ & Mesial & 9,68 & 1009 & 9,71 & 1000 \\
\hline & Distal & 10,49 & & 10,29 & \\
\hline$|\mathrm{mn}| 21$ & Mesial & 10,83 & 1120 & 10,46 & 1102 \\
\hline 1 & Distal & 11,56 & $11, \angle 0$ & 11,57 & 11,02 \\
\hline$|\mathrm{mp}| 22$ & Mesial & 11,82 & 1170 & 11,76 & 1167 \\
\hline 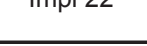 & Distal & 11,58 & 11,10 & 11,58 & 开, \\
\hline $1 \mathrm{~mol} 23$ & Mesial & 11,13 & 11160 & 11,08 & 1119 \\
\hline тाр 25 & Distal & 12,07 & 11,00 & 11,89 & 11,45 \\
\hline $\mid \mathrm{mnl} 24$ & Mesial & 10,92 & 10.47 & 10,83 & 1040 \\
\hline 1 Impl 24 & Distal & 10,01 & $10,4 I$ & 9,96 & 10,40 \\
\hline Impl 25 & Mesial & 10,41 & 10.52 & 10,03 & 1029 \\
\hline (1) & Distal & 10,63 & 10,02 & 10,54 & 10,20 \\
\hline
\end{tabular}


Tabla 3. Promedio de la diferencia del nivel óseo entre 24 y 30 meses.

\begin{tabular}{|c|c|c|c|c|c|}
\hline PACIENTE & Sitio & $\begin{array}{c}30 \\
\text { meses }\end{array}$ & $\begin{array}{c}24 \\
\text { meses }\end{array}$ & $\begin{array}{c}\text { Reab_ } \\
\text { ósea }\end{array}$ & $\begin{array}{l}\text { Prom } \\
\text { reab MD }\end{array}$ \\
\hline \multirow{2}{*}{ Impl 1} & Mesial & 11,08 & 11,07 & 0,01 & \multirow{2}{*}{0,05} \\
\hline & Distal & 10,97 & 10,88 & 0,09 & \\
\hline \multirow{2}{*}{ Impl 2} & Mesial & 11,72 & 11,68 & 0,04 & \multirow{2}{*}{0,08} \\
\hline & Distal & 11,53 & 11,41 & 0,12 & \\
\hline \multirow{2}{*}{ Impl 3} & Mesial & 9,19 & 9,16 & 0,03 & \multirow{2}{*}{0,09} \\
\hline & Distal & 10,18 & 10,04 & 0,14 & \\
\hline \multirow{2}{*}{ Impl 4} & Mesial & 10,24 & 10,17 & 0,07 & \multirow{2}{*}{0,05} \\
\hline & Distal & 9,95 & 9,93 & 0,02 & \\
\hline \multirow{2}{*}{ Impl 5} & Mesial & 10,73 & 10,66 & 0,07 & \multirow{2}{*}{0,11} \\
\hline & Distal & 10,42 & 10,28 & 0,14 & \\
\hline \multirow{2}{*}{ Impl 6} & Mesial & 12,13 & 12,08 & 0,05 & \multirow{2}{*}{0,06} \\
\hline & Distal & 13,10 & 13,03 & 0,07 & \\
\hline \multirow{2}{*}{ Impl 7} & Mesial & 10,75 & 10,58 & 0,17 & \multirow{2}{*}{0,09} \\
\hline & Distal & 11,15 & 11,14 & 0,01 & \\
\hline \multirow{2}{*}{ Impl 8} & Mesial & 13,21 & 12,85 & 0,36 & \multirow{2}{*}{0,36} \\
\hline & Distal & 13,21 & 12,85 & 0,36 & \\
\hline \multirow{2}{*}{ Impl 9} & Mesial & 11,03 & 11,03 & 0 & \multirow{2}{*}{0,04} \\
\hline & Distal & 10,97 & 10,89 & 0,08 & \\
\hline \multirow{2}{*}{ Impl 10} & Mesial & 12,64 & 12,58 & 0,06 & \multirow{2}{*}{0,19} \\
\hline & Distal & 12,95 & 12,63 & 0,32 & \\
\hline \multirow{2}{*}{ Impl 11} & Mesial & 11,37 & 11,36 & 0,01 & 010 \\
\hline & Distal & 9,65 & 9,42 & 0,23 & 0,12 \\
\hline Imn 12 & Mesial & 10,53 & 10,49 & 0,04 & -0.17 \\
\hline $1010 \mathrm{P}$ & Distal & 10,32 & 10,7 & $-0,38$ & $-0,11$ \\
\hline Impl 13 & Mesial & 11,05 & 11 & 0,05 & 016 \\
\hline 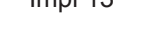 & Distal & 10,34 & 10,07 & 0,27 & 0,10 \\
\hline Impl 14 & Mesial & 10,51 & 10,53 & $-0,02$ & $0 \Omega 4$ \\
\hline mipr 14 & Distal & 10,9 & 10,8 & 0,1 & 0,07 \\
\hline Imn 15 & Mesial & 12,56 & 12,46 & 0,1 & O 07 \\
\hline 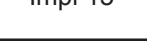 & Distal & 11,57 & 11,54 & 0,03 & 0,01 \\
\hline Impl 16 & Mesial & 9,65 & 9,23 & 0,42 & 2? \\
\hline & Distal & 9,90 & 9,89 & 0,01 & \\
\hline $\operatorname{Imnl} 17$ & Mesial & 10,35 & 10,01 & 0,34 & 0.34 \\
\hline & Distal & 10,39 & 10,05 & 0,34 & 0,34 \\
\hline 190 & Mesial & 10,36 & 10,09 & 0,27 & ק? \\
\hline $1 \mathrm{mpl} 18$ & Distal & 10,74 & 10,42 & 0,32 & 0,30 \\
\hline 10 & Mesial & 11,99 & 11,73 & 0,26 & 0 \\
\hline (19ир 19 & Distal & 9,18 & 9,13 & 0,05 & 0,10 \\
\hline 100 & Mesial & 9,68 & 9,71 & $-0,03$ & 000 \\
\hline . & Distal & 10,49 & 10,29 & 0,20 & 0,09 \\
\hline 101 & Mesial & 10,83 & 10,46 & 0,37 & 10 \\
\hline वारा 21 & Distal & 11,56 & 11,57 & $-0,01$ & 0,10 \\
\hline $\mid \mathrm{mnl} 22$ & Mesial & 11,82 & 11,76 & 0,06 & 003 \\
\hline & Distal & 11,58 & 11,58 & 0,00 & \\
\hline $1 \mathrm{~mol} 23$ & Mesial & 11,13 & 11,08 & 0,05 & 12 \\
\hline 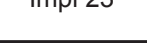 & Distal & 12,07 & 11,89 & 0,18 & 0,12 \\
\hline $1 \mathrm{mn} / 24$ & Mesial & 10,92 & 10,83 & 0,09 & 0707 \\
\hline 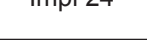 & Distal & 10,01 & 9,96 & 0,05 & , T, \\
\hline $1 \mathrm{mn} 25$ & Mesial & 10,41 & 10,03 & 0,38 & 024 \\
\hline (1) & Distal & 10,63 & 10,54 & 0,09 & 0,24 \\
\hline
\end{tabular}

Valores $<0$, ganancia ósea

Valores $>0$, pérdida ósea de construcción de la corona pilar no constituye un factor predisponente en el desarrollo de cuadros de perimplantitis.

Este análisis puede explicar la sobrevida del $100 \%$ de los implantes, ya que todos los implantes colocados luego de dos años y medio de su rehabilitación con prótesis removible parcial implanto-retenida aún permanecen en boca en condiciones funcionales.

Considerando los puntos evaluados en los párrafos anteriores, podemos señalar que esta nueva técnica de rehabilitación protésica es factible de realizar, además, según diversos estudios se ha demostrado que el uso de prótesis sigue en ascenso, producto del envejecimiento de la población; un gran número de personas todavía dependen de las prótesis dentales removibles para la función oral.

Al ser esta nueva técnica una alternativa de menor costo, se puede volver una opción favorable con mayor posibilidad de aplicarse en personas con recursos limitados, dado que ofrece una mejor estabilidad, comodidad y seguridad que las prótesis convencionales.

\section{CONCLUSIONES}

Los patrones de reabsorción ósea observados en la investigación realizada son similares a los que acontecen con otras formas de rehabilitación sobre implantes oseointegrados.

\section{Bibliografía}

1. Thalji G, McGraw K, Cooper LF. Maxillary Complete Denture Outcomes: A Systematic Review of Patient-Based Outcomes. Int J Oral Maxillofac Implants. 2016;31:169-81.

2. Komagamine Y, Kanazawa M, Kaiba Y, Sato Y, Minakuchi S, Sasaki Y. Association between self-assessment of complete dentures and oral health-related quality of life. J Oral Rehabil. 2012; Nov: 39(11):847-57.

3. Prithviraj DR, Madan V, Harshamayi P et al. A comparison of masticatory efficiency inconventional dentures, implant retained or supported overdentures and implant supported fixed prostheses: a literature review. J Dent Implant. 2014;4:153-7.

4. López CS, Saka CH, Rada G et al. Impact of fixed implant supported prostheses in edentulous patients: protocol for a systematic review. BMJ Open 2016;6:e009288. 5. INE, Chile: Proyecciones y Estimaciones de Población. www.ministeriodesarrollosocial. gob.cl/ipos/pdf/ipos_2011_poblacion.pdf.

6. Minsal, Guía Clínica Salud Oral Integral para adultos de 60 años, $2^{\circ}$ edición, 2010;1:10 [citado 24 mayo 2016]; Disponible en: http://web.minsal.cl/portal/url/item 17221747c2c9484b7e04001011f0141a4.pdf

7. Vogel R, Smith-Palmer J, Valentine W.Evaluating the health economic implications and cost-effectiveness of dental implants: a literature review. Int J Oral Maxillofac Implants. 2013 Mar-Apr;28(2):343-56.

8. Gholami H, Mericske-Stern R, Kessler-Liechti G et al. Radiographic Bone Level Changes of Implant-Supported Restorations in Edentulous and Partially Dentate Patients: 5-Year Results. Int J Oral Maxillofac Implants 2014;29(4):898-904.

9. Vercruyssen M, Marcelis K, Coucke W et al. Long-term, retrospective evaluation (implant and patient-centre outcome) of the two-implants-supported overdenture in the mandible. Part 1: marginal bone loss. Clin Oral Impl Res. 2010;21:466-72.

10. Elie E. Daou. Stud attachments for the mandibular implant-retained overdentures: Prosthetic complications. A literature review. Saudi Dent J. 2013 Apr; 25(2): 53-60. 11. Mahmoud Darwish, Mohammad Zakaria Nassani, Kusai Baroudi. Effect of neutral zone technique on marginal bone loss around implant-supported overdentures. J Int Soc Prev Community Dent. 2015 Dec; 5(Suppl 2): 57-S62. 